\title{
A Necessary and Sufficient Minimality Condition for Uncertain Systems
}

\author{
Carolyn L. Beck, Associate Member, IEEE and John Doyle, Member, IEEE
}

\begin{abstract}
A necessary and sufficient condition is given for the exact reduction of systems modeled by linear fractional transformations (LFT's) on structured operator sets. This condition is based on the existence of a rank-deficient solution to either of a pair of linear matrix inequalities which generalize Lyapunov equations; the notion of Gramians is thus also generalized to uncertain systems, as well as Kalman-like decomposition structures. A related minimality condition, the converse of the reducibility condition, may then be inferred from these results and the equivalence class of all minimal LFT realizations defined. These results comprise the first stage of a complete generalization of realization theory concepts to uncertain systems. Subsequent results, such as the definition of and rank tests on structured controllability and observability matrices are also given. The minimality results described herein are applicable to multidimensional system realizations as well as to uncertain systems; connections to formal powers series representations also exist.
\end{abstract}

Index Terms-Minimality, model reduction, uncertain systems.

\section{INTRODUCTION}

A STANDARD framework for studying uncertain systems uses structured perturbations on a nominal model, with linear fractional transformations (LFT's) as the basic realization tool. This framework was introduced explicitly almost 20 years ago [1]-[3], although it was implicit in much earlier work in control theory. A comprehensive theory of system analysis and synthesis has been developed in this framework, involving a great variety of assumptions on the uncertainty (see, for example, [4]-[11] and the references therein). Recently, a state-space theory has begun to emerge for uncertain systems represented by LFT's on structured uncertainty operators, which we will refer to as uncertain LFT systems. This allows for the finite dimensionality of the realizations to be exploited, and analysis tests and design constructs can be developed in terms of finite-dimensional constant matrices, usually in terms of linear matrix inequalities (LMI's). For example, stability and $l_{2}$ gain may be characterized in terms of generalized Lyapunov inequalities [12], balanced model reduction methods with guaranteed error bounds may be stated [13], [14], and an output feedback stabilization method using a separation argument may be given [15].

Manuscript received May 9, 1997; revised April 10, 1998. Recommended by Associate Editor, J. Chen.

C. L. Beck is with the Department of General Engineering, University of Illinois at Urbana-Champaign, Urbana, IL 61801 USA.

J. Doyle is with Control and Dynamical Systems, California Institute of Technology, Pasadena, CA 91125 USA.

Publisher Item Identifier S 0018-9286(99)07843-5.
In this paper, the notion of reducibility and the converse notion of minimality for uncertain LFT systems are discussed in detail. Exact reducibility of uncertain systems realizations is shown to be equivalent to the existence of a rank-deficient solution to either of a pair of LMI's. This LMI condition directly extends the model reduction results given in [14] to the case of model reduction with no error, but substantially new and different proof techniques are needed. The earlier work used well-known LMI machinery for LFT's [7] which do not extend to the exact case. Perhaps more importantly, the results in this paper also directly relate to more pure realization theory concepts for uncertain systems, such as the development of controllability and observability matrices, the decomposition of the system variable space into reachable and unobservable subspaces, and to the construction of Kalmanlike decomposition structures, as well as to formal power series results of the 1970's. Structured controllability and observability matrices for uncertain systems are defined herein, and the connections between rank deficiencies of these matrices and the reducibility results are discussed. The construction of Kalman-like decomposition structures for uncertain systems are also reviewed in this setting, leading to a complete generalization of minimality for uncertain systems via an algebraic approach. Preliminary presentations of the minimality results presented in this paper were first given in [16] and [17]. The controllability and observability structures were first defined in [13] and presented in [18] in conjunction with a discussion of related reachable and unobservable system subspaces. Preliminary computational findings for algorithms based on these reducibility results may be found in [19] and [20].

This paper is organized as follows: we first introduce our notation and give a brief review of the general LFT framework in Section II. In Section III, introductory realization theory for uncertain systems is discussed, including equivalence of realizations, stability and Lyapunov equations, and Gramians. The main result of this paper is the necessary and sufficient reducibility condition given in Section IV; a minimality result is also stated, which defines as an equivalence class all minimal LFT realizations. Related Kalman-like decomposition structures are presented in Section V, along with a discussion on structured controllability and observability matrices. Conclusions are given in Section VI and include a brief discussion of related state-space results for uncertain systems.

\section{PRELIMINARIES}

The notation we use is as follows: $l_{2}$ denotes the space of sequences which are square summable, and $\mathcal{L}\left(l_{2}\right)$ represents 


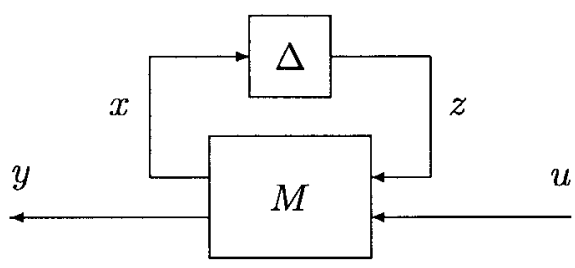

Fig. 1. LFT/uncertain system.

the space of all linear operators on $l_{2}$. We represent the group of matrices in the real and complex fields by $\mathbf{R}^{n \times m}$ and $\mathbf{C}^{n \times m}$. The shift operator on $l_{2}$ is denoted by $\boldsymbol{\lambda}$, and the identity matrix is denoted by $I$. The maximum singular value of $A \in \mathbf{C}^{n \times m}$ is denoted by $\bar{\sigma}(A) ; A^{*}$ denotes the complex conjugate transpose. The dimensions of a matrix $A$ are denoted $\operatorname{dim}(A)$. For notational convenience, dimensions will not be given unless pertinent to the discussion.

We consider uncertain dynamic systems evolving in discrete time, with uncertainty described by structured linear timevarying (LTV) operators on $l_{2}$; this last assumption is not strictly necessary and it can be equivalently assumed that the uncertainty consists simply of noncommuting indeterminants.

\section{A. LFT's}

The main focus of robust control has been to evaluate the effects of uncertainty-for example noise, disturbances, parameter variations, nondominant nonlinearities, and unmodeled dynamics - when analyzing and designing controllers for dominantly linear systems. Over the past decade, the LFT paradigm has been widely used as a mathematical representation for uncertainty in system models. This paradigm is represented pictorially in Fig. 1 and described below.

In much of the robust control literature, $M$ represents the nominal system model consisting of a linear time-invariant transfer function for the plant plus system weighting functions on the inputs and outputs, and $\Delta$ represents the uncertainty. In the LFT models we consider, the shift operator $\lambda$ corresponding to the system transform variable is also included in $\Delta$; that is, the transfer functions for the plant and weightings are explicitly written as LFT's on the shift operator. Thus without loss in generality, we may assume $M$ is a constant matrix, and $\Delta$ represents the system uncertainty and shift operators. In particular, we refer to $M$ as the system realization matrix, which we partition as

$$
M=\left[\begin{array}{ll}
A & B \\
C & D
\end{array}\right]
$$

and we assume $\Delta$ lies in some prescribed set.

Because each perturbation source is likely to enter the real system at a different location, the resulting structure for $\Delta$ is block diagonal (see [4] and [5] for further details and examples). The uncertainty set $\boldsymbol{\Delta}$ is thus defined by

$$
\begin{aligned}
\Delta:= & \left\{\operatorname{diag}\left[\delta_{1} I_{n_{1}}, \cdots, \delta_{p} I_{n_{p}}, \Delta_{m_{1}}, \cdots, \Delta_{m_{f}}\right]:\right. \\
& \left.\delta_{i} \in \mathcal{L}\left(l_{2}\right), \quad \Delta_{m_{i}} \in \mathcal{L}\left(l_{2}^{m_{i}}\right)\right\} .
\end{aligned}
$$

We refer to the $\delta_{i} I_{n_{i}}$ as repeated scalar blocks and to the $\Delta_{j}$ as full blocks. The results discussed in this paper are necessary and sufficient for uncertainty modeled by LTV operators on $l_{2}$ and are sufficient when an additional structure such as real parametric variance or time-invariance is imposed, for example, if $\boldsymbol{\Delta}$ represents multiple transform variables in a multidimensional system. For analysis purposes we will often consider $\Delta$ which lie in a norm-bounded subset of $\boldsymbol{\Delta}$, that is

$$
\mathbf{B}_{\Delta}=\left\{\Delta \in \Delta:\|\Delta\|_{l_{2} \rightarrow l_{2}} \leq 1\right\}
$$

where $\|\cdot\|_{l_{2} \rightarrow l_{2}}$ denotes the induced norm. We will denote these uncertain system models by the pair $(\boldsymbol{\Delta}, M)$.

The input/output ( $\mathrm{I} / \mathrm{O})$ mapping from $u$ to $y$ is given by the LFT

$$
y=(\Delta \star M) u, \quad \Delta \in \Delta
$$

where

$$
\Delta \star M:=D+C \Delta(I-A \Delta)^{-1} B
$$

whenever the inverse is well-defined. We assume throughout the sequel that $y \in l_{2}^{q}$ and $u \in l_{2}^{m}$, although this assumption is not required for the results in this paper. For notational convenience, dimensions will not be given in the sequel unless required for clarity.

\section{B. Repeated Scalar Uncertainty Structures}

In this paper, we focus on repeated scalar uncertainty sets, that is

$$
\boldsymbol{\Delta}=\left[\begin{array}{llll}
\delta_{1} I_{n_{1}} & & & \\
& \delta_{2} I_{n_{2}} & & \\
& & \ddots & \\
& & & \delta_{p} I_{n_{p}}
\end{array}\right]
$$

where one of the $\delta_{i}$ represents $\boldsymbol{\lambda}$. Although many of the results we present are valid for the uncertainty structure given in (1), with both repeated scalar and full uncertainty blocks, for the reducibility results described herein the repeated scalar case is the more technically interesting case, as well as notationally the cleaner case.

As we often consider inputs and outputs as signals in $l_{2}$, we may consider the $\delta_{i}$ to be arbitrary time-varying operators on $l_{2}$. Alternatively, we may assume the $\delta_{i}$ represent real-valued parametric uncertainty, component tolerances for example, or we may assume the $\delta_{i}$ are used to represent a multidimensional system. Generally speaking, the more structure that is imposed on the set $\boldsymbol{\Delta}$, the more difficult computation for analysis and design becomes. If the only structure we assume for the uncertainty is spatial, then the LFT of a matrix $M$ on $\boldsymbol{\Delta}$ reduces to a representation of rational functions in multiple noncommuting indeterminates. Such an LFT system may then be viewed as a particular realization of a formal power series [13], [16], [21].

For most of the results discussed in this paper we assume the $\delta_{i}$ are noncommuting variables, be they either completely abstract indeterminants in a power series or arbitrary timevarying operators on $l_{2}$. The results we obtain are then applicable to all of the aforementioned cases, to the more abstract settings as well as to systems with parametric uncertainty or multidimensional systems; in the latter cases this may lead to conservative conditions. 


\section{INTRODUCTORY REALIZATION \\ THEORY FOR UNCERTAIN SYSTEMS}

Analogous to the standard state-space framework, given an LFT realization $(\boldsymbol{\Delta}, M)$, one way to obtain an equivalent realization is by applying a structured similarity transformation. Furthermore, the existence of structured positive definite solutions to system LMI's, in particular to Lyapunov inequalities, is equivalent to $l_{2}$-stability of the system. These Lyapunov inequality solutions also lead to a notion of structured Gramians for uncertain systems, which in conjunction with the similarity transformations lead to a notion of balanced realizations for uncertain systems. We describe these constructs and the significance of the structure required in this section; for full details see [13] and [14].

\section{A. Equivalent Realizations}

For repeated scalar uncertainty structures, we define equivalence as follows.

Definition 1: Two realizations

$$
\begin{aligned}
& \boldsymbol{\Delta}_{1}=\left\{\left[\begin{array}{lll}
\delta_{1} I_{n_{1}} & & \\
& \ddots & \\
& & \delta_{p} I_{n_{p}}
\end{array}\right]: \delta_{i} \in \mathcal{L}\left(l_{2}\right)\right\} \\
& M_{1}=\left[\begin{array}{cc}
A_{1} & B_{1} \\
C_{1} & D
\end{array}\right]
\end{aligned}
$$

and

$$
\begin{aligned}
\boldsymbol{\Delta}_{2} & =\left\{\left[\begin{array}{lll}
\delta_{1} I_{r_{1}} & & \\
& \ddots & \\
& & \delta_{p} I_{r_{p}}
\end{array}\right]: \delta_{i} \in \mathcal{L}\left(l_{2}\right)\right\} \\
M_{2} & =\left[\begin{array}{cc}
A_{2} & B_{2} \\
C_{2} & D
\end{array}\right]
\end{aligned}
$$

are equivalent if $\Delta_{1} \star M_{1}=\Delta_{2} \star M_{2}$ for all $\delta_{i} \in \mathcal{L}\left(l_{2}\right)$, $i=1, \cdots, p$.

Note that $\Delta_{2}$ is constructed using the same uncertainty variables as is $\Delta_{1}$, but with possibly different dimensions.

Similarity transformations are defined for LFT realizations as in the standard case and provide one method for easily obtaining an alternative but equivalent realization. However, in general, for a transformed realization of an uncertain system to be equivalent to the original realization, the transformation must commute with the uncertainty structure.

Definition 2: Let $n=\sum_{i=1}^{p} n_{i}+\sum_{j=1}^{f} m_{j}$. The commutative matrix set for a given uncertainty set $\boldsymbol{\Delta}$ is denoted by $\mathcal{T}$ and defined by

$$
\mathcal{T}:=\left\{T \in \mathbf{C}^{n \times n}: T \Delta=\Delta T, \quad \text { for all } \Delta \in \boldsymbol{\Delta}\right\} .
$$

When $\boldsymbol{\Delta}$ is defined as in (1), the set $\mathcal{T}$ has the block diagonal structure

$$
\begin{array}{r}
T=\operatorname{diag}\left[T_{1}, \cdots, T_{p}, t_{1} I_{m_{1}}, \cdots, t_{f} I_{m_{f}}\right], \\
\text { where each } T_{i} \in \mathbf{C}^{n_{i} \times n_{i}} \text { and } t_{j} \in \mathbf{C} .
\end{array}
$$

We refer to a nonsingular element $T$ in the set $\mathcal{T}$ as an allowable transformation. Given an LFT realization $(\boldsymbol{\Delta}, M)$ and any nonsingular $T \in \mathcal{T}$, it is straightforward to show using (3) that an equivalent LFT system is defined by the realization matrices $\left\{T A T^{-1}, T B, C T^{-1} D\right\}$. Note that permutations of both the uncertainty variables and the realization matrices are also allowed, as neither the resulting I/O mapping nor the norm bound of the uncertainty is affected.

A truncation of the realization matrices may also produce an equivalent realization, in which case the original realization $(\boldsymbol{\Delta}, M)$ is said to be reducible. Reducibility is discussed in this paper in terms of the existence of rank deficient matrix solutions to a pair of Lyapunov inequalities. As with standard state-space realizations, it is generally assumed that the LFT realizations are stable when evaluating the system Lyapunov inequalities for the existence of solutions.

\section{B. Lyapunov Inequalities and Stability}

Consider the system in Fig. 1 with $\boldsymbol{\Delta}$ and $M$ defined as in (1). We say such a system is stable when the map $(\Delta \star M)$ is well defined for every $\Delta \in \mathbf{B}_{\Delta}$; precisely speaking, this is a robust $l_{2}$-stability condition which we will henceforth refer to simply as stability.

Definition 3: Let $M=\left[\begin{array}{ll}A & C \\ B & D\end{array}\right]$ be a constant matrix and $\boldsymbol{\Delta} \subset \mathcal{L}\left(l_{2}\right)$. The system defined by the pair $(\boldsymbol{\Delta}, M)$ is stable if $(I-A \Delta)$ is invertible in $\mathcal{L}\left(l_{2}\right)$ for each $\Delta \in \mathbf{B}_{\Delta}$.

If the only assumption placed on the uncertainty set $\Delta$ is the spatial structure, that is, $\Delta$ consists of full block and repeated scalar block structured linear operators on $l_{2}$, then a necessary and sufficient LMI stability condition has been found which is stated below in Theorem 4. Details can be found in [22]. This condition extends the sufficient scaled small gain condition for robust stability and results on the necessity of constant scalings for LTV uncertainty obtained independently by Megretski [11], [23] and by Shamma [8] for full block diagonal uncertainty structures. Note that one of the $\delta_{i}$ may represent the shift operator $\lambda$.

Theorem 4 [22]: Given an uncertainty set, $\Delta \subset \mathcal{L}\left(l_{2}\right)$, and a constant matrix $A$

$$
(I-A \Delta) \text { is invertible in } \mathcal{L}\left(l_{2}\right), \quad \text { for all } \Delta \in \mathbf{B}_{\Delta}
$$

if and only if there exists a matrix $Y>0, Y \in \mathcal{T}$ such that

$$
A Y A^{*}-Y<0 .
$$

Since this stability condition is defined in terms of $A$ and $\Delta$, we will sometimes say the matrix $A$ is stable with respect to the $\Delta$ structure, meaning that the map $(\Delta \star M)$ is well defined for all $\Delta \in \mathbf{B}_{\Delta}$.

The LMI condition in (5) directly extends the Lyapunov inequality test for stability of a standard state-space system. Using the LMI condition of (5), we can readily show the following (see also [15, Th. 3.8]), which is used in the proof of the reducibility condition in Section IV.

Lemma 5: Given a constant matrix $A$ with an associated uncertainty structure $\Delta$, where

$$
A=\left[\begin{array}{cc}
A_{11} & A_{12} \\
0 & A_{22}
\end{array}\right] \text { and } \Delta=\left[\begin{array}{cc}
\Delta_{1} & 0 \\
0 & \Delta_{2}
\end{array}\right]
$$

then there exists a matrix $Y>0, Y \in \mathcal{T}$ satisfying $A Y A^{*}-$ $Y<0$, if and only if there exist matrices $Y_{1}>0, Y_{1} \in \mathcal{T}_{1}$, 
and $Y_{2}>0, Y_{2} \in \mathcal{T}_{2}$ satisfying

$$
A_{11} Y_{1} A_{11}^{*}-Y_{1}<0 \quad \text { and } A_{22} Y_{2} A_{22}^{*}-Y_{2}<0 .
$$

Proof: See the Appendix.

Remark 6: For systems that are modeled using real or complex-valued uncertainty, as well as multidimensional systems, the stability condition given in Theorem 4 is sufficient, but not necessary. For these systems, structured singular value conditions lead to both necessary and sufficient stability criteria [10]. Results in [24] and [25] have shown that the computational problems associated with the structured singular value in these cases is NP-hard. The condition stated in Theorem 4 is computable via convex optimization techniques.

\section{Structured Gramians}

An obviously equivalent stability condition to the LMI condition in (5) is the existence of a matrix $X>0, X \in$ $\mathcal{T}$ satisfying $A^{*} X A-X<0$. By scaling $Y$ and $X$ by constant gains, we immediately obtain the following corollary to Theorem 4, generalizing the notion of Lyapunov equations for standard system realizations.

Corollary 7: If $(\boldsymbol{\Delta}, M)$ is stable, then there exist $Y \geq 0$ and $X \geq 0$, both in $\mathcal{T}$, which satisfy the Lyapunov inequalities

$$
A Y A^{*}-Y+B B^{*} \leq 0 \text { and } A^{*} X A-X+C^{*} C \leq 0 \text {. (6) }
$$

We refer to any matrices $Y \geq 0$ and $X \geq 0$ in $\mathcal{T}$ that satisfy (6) as structured Gramians. Structured Gramians may be computed using convex programming methods specifically developed for solving LMI's (see, for example, [26] and [27]). Unlike true Gramians, structured Gramians are not unique as they are not solutions to the system Lyapunov equations.

As in the standard case, balanced realizations may also be constructed for uncertain systems by finding an allowable transformation $T$ that simultaneously diagonalizes the structured Gramians $Y$ and $X$; for details see [14] and references therein.

Note that the LMI's in (5) and (6) are not affected by permutations of the realization matrices $A, B$, and $C$ and the uncertainty structure $\Delta$. The following lemma is easily shown using such permutations.

Lemma 8: Suppose

$$
A=\left[\begin{array}{cc|cc}
A_{11} & A_{12} & A_{13} & A_{14} \\
0 & A_{22} & 0 & A_{24} \\
\hline A_{31} & A_{32} & A_{33} & A_{34} \\
0 & A_{42} & 0 & A_{44}
\end{array}\right]
$$

is stable with respect to the uncertainty structure

$$
\Delta=\left[\begin{array}{c|c}
\delta_{1} I_{n_{1}} & 0 \\
\hline 0 & \delta_{2} I_{n_{2}}
\end{array}\right] .
$$

Then

$$
\left[\begin{array}{l|l}
A_{22} & A_{24} \\
\hline A_{42} & A_{44}
\end{array}\right]
$$

is stable with respect to the structure

$$
\Delta_{r}=\left[\begin{array}{c|c}
\delta_{1} I_{r_{1}} & 0 \\
\hline 0 & \delta_{2} I_{r_{2}}
\end{array}\right]
$$

where $r_{1}=\operatorname{dim}\left(A_{22}\right)$ and $r_{2}=\operatorname{dim}\left(A_{44}\right)$.

Similarly, we can show that

$$
\left[\begin{array}{l|l}
A_{11} & A_{13} \\
\hline A_{31} & A_{33}
\end{array}\right]
$$

is stable with respect to the uncertainty structure

$$
\Delta_{r}=\left[\begin{array}{c|c}
\delta_{1} I_{q_{1}} & 0 \\
\hline 0 & \delta_{2} I_{q_{2}}
\end{array}\right]
$$

where $r_{1}+q_{1}=n_{1}$ and $r_{2}+q_{2}=n_{2}$.

\section{A NeCESSARY AND SufFicient Reducibility Condition}

For standard one-dimensional (1-D) systems there is a welldefined notion of minimality, or equivalently controllability and observability. In order to develop similar definitions for system models which incorporate uncertainty descriptions into the realizations, we first prove the following sufficient condition for exact reducibility, stated in Theorem 9. This condition provides the first step in the development of realization theory results for uncertain systems and is valid for $\delta_{i}$ representing transform variables, norm-bounded real or complex perturbations, or time-varying operators on $l_{2}$, thus, this result is applicable to both multidimensional and uncertain system realizations. It should be noted that although the reducibility results presented in this section look similar in statement to the model reduction results of [14], a totally different approach is required to prove the exact reducibility case; a limit-based argument applied to the results of [14], or [7] for that matter, will not lead to a proper solution for the Lyapunov inequalities under consideration.

Throughout this section we denote the full and reduced system realizations by

$$
M=\left[\begin{array}{ll}
A & B \\
C & D
\end{array}\right] \text { and } \quad M_{r}=\left[\begin{array}{cc}
A_{r} & B_{r} \\
C_{r} & D
\end{array}\right]
$$

with corresponding repeated scalar uncertainty structures

$$
\boldsymbol{\Delta}=\left\{\operatorname{diag}\left[\delta_{1} I_{n_{1}}, \delta_{2} I_{n_{2}}, \cdots, \delta_{p} I_{n_{p}}\right]: \delta_{i} \in \mathcal{L}\left(l_{2}\right)\right\}
$$

and

$$
\begin{aligned}
\boldsymbol{\Delta}_{r} & =\mathcal{I}_{r}(\boldsymbol{\Delta}) \\
& =\left\{\operatorname{diag}\left[\delta_{1} I_{r_{1}}, \delta_{2} I_{r_{2}}, \cdots, \delta_{p} I_{r_{p}}\right]: \delta_{i} \in \mathcal{L}\left(l_{2}\right)\right\}
\end{aligned}
$$

where the notation $\mathcal{I}_{r}(\boldsymbol{\Delta})$ is used to emphasize that $\Delta_{r}$ represents a reduced or lower dimension copy of $\Delta$ and is not an independent uncertainty structure. The difference between the full and reduced realizations, $(\Delta \star M)-\left(\Delta_{r} \star M_{r}\right)$, is realized by

$$
\tilde{E}=\left[\begin{array}{ccc}
A & 0 & B \\
0 & A_{r} & B_{r} \\
C & -C_{r} & 0
\end{array}\right]
$$


and

$$
\tilde{\boldsymbol{\Delta}}=\left\{\left[\begin{array}{cc}
\Delta & 0 \\
0 & \Delta_{r}
\end{array}\right]: \Delta \in \Delta, \Delta_{r} \in \Delta_{r}\right\} .
$$

The full and reduced realizations are equivalent if and only if $\tilde{\Delta} \star \tilde{E}=0$ for all $\tilde{\Delta} \in \tilde{\Delta}$.

Note that as a result of the dependence between $\boldsymbol{\Delta}$ and $\boldsymbol{\Delta}_{r}$, the commutative matrix set for $\tilde{\boldsymbol{\Delta}}$ includes matrices with the following block structure:

$$
T=\left[\begin{array}{cc}
\operatorname{diag}\left(T_{i}^{n}\right) & \operatorname{diag}\left(T_{i}^{n r}\right) \\
\operatorname{diag}\left(T_{i}^{r n}\right) & \operatorname{diag}\left(T_{i}^{r}\right)
\end{array}\right]
$$

where $\operatorname{dim}\left(T_{i}^{n}\right)=n_{i} \times n_{i}, \operatorname{dim}\left(T_{i}^{n r}\right)=n_{i} \times r_{i}, \operatorname{dim}\left(T_{i}^{r n}\right)=$ $r_{i} \times n_{i}$, and $\operatorname{dim}\left(T_{i}^{r}\right)=r_{i} \times r_{i}$ for all $i=1, \cdots, p$.

Theorem 9-Sufficiency: Given the stable system representation $(\boldsymbol{\Delta}, M)$, there exists a reduced representation, $\left(\boldsymbol{\Delta}_{r}, M_{r}\right)$, such that $(\tilde{\Delta} \star \tilde{E})=0$ for all $\tilde{\Delta} \in \mathbf{B}_{\tilde{\Delta}}$, if there exists singular $X \geq 0$ or $Y \geq 0$, both in $\mathcal{T}$, satisfying:

1) $A Y A^{*}-Y+B B^{*} \leq 0$;

2) $A^{*} X A-X+C^{*} C \leq 0$.

Furthermore, $\max \left(\operatorname{dim}(\Delta)-\operatorname{dim}\left(\Delta_{r}\right)\right)$ is equal to the number of zero-valued eigenvalues of the product $Y X$.

Proof: Suppose there exists $Y \geq 0$ satisfying 1). (The proof for $X \geq 0$ satisfying 2) is essentially the same, and therefore is not presented.)

Without loss of generality, we can assume $p=2$, that is, that $\boldsymbol{\Delta}=\left\{\operatorname{diag}\left[\delta_{1} I_{n_{1}}, \delta_{2} I_{n_{2}}\right]: \delta_{i} \in \mathcal{L}\left(l_{2}\right)\right\}$. The proof extends immediately to $p>2$, either directly or by recursive application.

Suppose $Y=\left[\begin{array}{cc}Y_{1} & 0 \\ 0 & Y_{2}\end{array}\right]$ with $Y_{1}>0$ and $Y_{2} \geq 0$, where $Y_{i}$ has the same dimensions as $I_{n_{i}}, i=1,2$. (If $Y_{1} \geq 0$, the proof is the essentially the same, but notationally more cumbersome.) Furthermore, we can transform $Y_{2}$ to $\left[\begin{array}{cc}\hat{Y}_{2} & 0 \\ 0 & 0\end{array}\right]$ with $\hat{Y}_{2}>0$. We thus can assume $Y_{2}$ has this structure.

Partition the system matrices accordingly with respect to the structure of $Y$, that is

and

$$
A=\left[\begin{array}{ccc}
A_{1} & A_{12} & A_{13} \\
A_{21} & A_{22} & A_{23} \\
A_{31} & A_{32} & A_{33}
\end{array}\right], \quad B=\left[\begin{array}{l}
B_{1} \\
B_{2} \\
B_{3}
\end{array}\right]
$$

$$
C=\left[\begin{array}{lll}
C_{1} & C_{2} & C_{3}
\end{array}\right]
$$

where $A_{1}, B_{1}$, and $C_{1}$ are dimensioned compatibly with $Y_{1} ; A_{22}, B_{2}$, and $C_{2}$ are dimensioned compatibly with $\hat{Y}_{2}$; and $A_{33}, B_{3}$, and $C_{3}$ are dimensioned compatibly with the zero submatrix of $Y_{2}$. Partition $\Delta \in \Delta$ similarly so that $\Delta=\operatorname{diag}\left[\delta_{1} I_{n_{1}}, \delta_{2} I_{r_{2}}, \delta_{2} I_{q_{2}}\right]$, where $r_{2}+q_{2}=n_{2}$, and $r_{2}$ is the dimension of $\hat{Y}_{2}$.

By assumption, $A Y A^{*}-Y+B B^{*} \leq 0$. In particular

$$
\left[\begin{array}{lll}
A_{31} & A_{32} & A_{33}
\end{array}\right]\left[\begin{array}{ccc}
Y_{1} & 0 & 0 \\
0 & Y_{2}^{1} & 0 \\
0 & 0 & 0
\end{array}\right]\left[\begin{array}{c}
A_{31}^{*} \\
A_{32}^{*} \\
A_{33}^{*}
\end{array}\right]+B_{3} B_{3}^{*} \leq 0
$$

thus $A_{31}=0, A_{32}=0$, and $B_{3}=0$, since both $Y_{1}>0$ and $\hat{Y}_{2}>0$. Denote

$$
\begin{aligned}
& \tilde{A}_{11}=\left[\begin{array}{ll}
A_{1} & A_{12} \\
A_{21} & A_{22}
\end{array}\right], \quad \tilde{A}_{12}=\left[\begin{array}{l}
A_{13} \\
A_{23}
\end{array}\right] \\
& \tilde{A}_{22}=A_{33}, \quad \tilde{B}_{1}=\left[\begin{array}{l}
B_{1} \\
B_{2}
\end{array}\right], \quad \tilde{C}_{1}=\left[\begin{array}{ll}
C_{1} & C_{2}
\end{array}\right]
\end{aligned}
$$

and $\tilde{C}_{2}=C_{3}$.

Let

and

$$
M_{r}=\left[\begin{array}{cc}
\tilde{A}_{11} & \tilde{B}_{1} \\
\tilde{C}_{1} & D
\end{array}\right]
$$

$$
\Delta_{r}=\left\{\operatorname{diag}\left[\delta_{1} I_{n_{1}}, \delta_{2} I_{r_{2}}\right]: \delta_{i} \in \mathcal{L}\left(l_{2}\right)\right\} .
$$

Construct the difference realization $(\tilde{\Delta} \star \tilde{E})=(\Delta \star M)-$ $\left(\Delta_{r} \star M_{r}\right)$, and use the similarity transformation

$$
T_{0}=\left[\begin{array}{ccc}
I_{n_{1}+r_{2}} & 0 & -I_{n_{1}+r_{2}} \\
0 & 0 & I_{n_{1}+r_{2}} \\
0 & I_{q_{2}} & 0
\end{array}\right]
$$

so that the transformed difference system realization is

$$
\tilde{E}=\left[\begin{array}{cccc}
\tilde{A}_{11} & 0 & \tilde{A}_{12} & 0 \\
0 & \tilde{A}_{11} & 0 & \tilde{B}_{1} \\
0 & 0 & \tilde{A}_{22} & 0 \\
\tilde{C}_{1} & 0 & \tilde{C}_{2} & 0
\end{array}\right]
$$

and

$$
\tilde{\boldsymbol{\Delta}}=\left\{\operatorname{diag}\left[\Delta_{r}, \Delta_{r}, \delta_{2} I_{q_{2}}\right], \Delta_{r} \in \boldsymbol{\Delta}_{r}, \delta_{2} \in \mathcal{L}\left(l_{2}\right)\right\} .
$$

In order to show $(\tilde{\Delta} \star \tilde{E})=(\Delta \star M)-\left(\Delta_{r} \star M_{r}\right)=0$, we must first show both $\tilde{A}_{11}$ and $\tilde{A}_{22}$ are stable.

By assumption

$$
A=\left[\begin{array}{ccc}
A_{1} & A_{12} & A_{13} \\
A_{21} & A_{22} & A_{23} \\
0 & 0 & A_{33}
\end{array}\right]
$$

is stable with respect to the uncertainty set $\boldsymbol{\Delta}$, thus there exists

$$
Q=\left[\begin{array}{ccc}
Q_{1} & 0 & 0 \\
0 & Q_{22} & Q_{23} \\
0 & Q_{23}^{*} & Q_{33}
\end{array}\right]>0: A^{*} Q A-Q<0 .
$$

By extracting the upper left submatrix of (9) we see that $\tilde{A}_{11}^{*} \tilde{Q} \tilde{A}_{11}-\tilde{Q}<0$, where $\tilde{Q}=\operatorname{diag}\left[Q_{1}, Q_{22}\right]$. Therefore, $\tilde{A}_{11}$ is stable with respect to the uncertainty set $\boldsymbol{\Delta}_{r}$.

Similarly, we can show that $\tilde{A}_{22}$ is stable with respect to the uncertainty structure $\delta_{2} I_{q 2}$ by considering the lower right submatrix of the matrix inequality $A P A^{*}-P<0$, where a solution

$$
P=\left[\begin{array}{ccc}
P_{1} & 0 & 0 \\
0 & P_{22} & P_{23} \\
0 & P_{23}^{*} & P_{33}
\end{array}\right]>0
$$

exists, also by stability of $A$.

Since both $\tilde{A}_{11}$ and $\tilde{A}_{22}$ are stable, $\tilde{E}$ is stable with respect to the uncertainty set $\tilde{\Delta}$ by Lemma 5 . A straightforward calculation then shows $\tilde{\Delta} \star \tilde{E}=0$ for all $\tilde{\Delta} \in \mathbf{B}_{\tilde{\Delta}}$. 
The final statement of the theorem on the dimensions of the reduction can be seen directly from the constructions used to obtain $\left(\boldsymbol{\Delta}_{r}, M_{r}\right)$. In the case where both $X$ and $Y$ are singular, this is more easily seen by considering the allowable similarity transformation that results from simultaneously diagonalizing $X$ and $Y$.

Remark 10: If we consider reducibility of realizations with uncertainty structures containing full blocks, that is, $\Delta$ as defined in (1), then the submatrices $Y_{j}$ and $X_{j}$ of $Y$ and $X$, respectively, corresponding to the full blocks, $\Delta_{j}$, are diagonal scaling blocks, for example, $Y_{j}=y_{j} I_{m_{j}}, y_{j} \in \mathbf{R}$. Clearly, if $Y_{j}$ is singular, $y_{j}=0$. In this case, in order for the Lyapunov inequalities to hold, entire subblocks of the realization matrices $A, B$, and $C$ must be zero and the result is obvious.

Proving that the existence of a singular structured Gramian is also necessary for a lower dimension realization to exist gives us a complete notion of reducibility for uncertain systems which is similar to that for 1-D stable systems. The proof for the necessity condition is based on the following two lemmas; note that the assumption that the set $\Delta$ consists of noncommutative elements, $\delta_{i}$, is used. Thus this condition is not necessary for multidimensional systems, or systems with real or complex-valued time-invariant perturbations.

In the proof of Lemma 11, given in the Appendix, it is implicitly assumed that the uncertainty structure is $\boldsymbol{\Delta}=\left\{\operatorname{diag}\left[\delta_{1} I_{n_{1}}, \cdots, \delta_{p} I_{n_{p}}\right]\right\}$. The result can immediately be extended to uncertainty structures such as $\tilde{\boldsymbol{\Delta}}=$ $\left\{\operatorname{diag}\left[\delta_{1} I_{n_{1}}, \cdots, \quad \delta_{p} I_{n_{p}}, \delta_{1} I_{r_{1}}, \cdots, \delta_{p} I_{r_{p}}\right]\right\}$ by permuting $\tilde{\Delta}$ to

$$
\hat{\boldsymbol{\Delta}}=\left\{\operatorname{diag}\left[\delta_{1} I_{n_{1}+r_{1}}, \cdots, \delta_{p} I_{n_{p}+r_{p}}\right]\right\}
$$

and also permuting $\tilde{E}, Y$, and $X$ accordingly.

Lemma 11: Suppose the stable system realization $(\boldsymbol{\Delta}, M)$ is given, where $\boldsymbol{\Delta} \subset \mathcal{L}\left(l_{2}\right)$ is an arbitrary linear operator. If $(\Delta \star M)=0$, for all $\Delta \in \mathbf{B}_{\Delta}$, then there exist $X \geq 0$ and $Y \geq 0$, both in $\mathcal{T}$, satisfying

1) $A Y A^{*}-Y+B B^{*} \leq 0$;

2) $A^{*} X A-X+C^{*} C \leq 0$;

3) $X Y=0$.

Proof: See the Appendix.

This proof relies on expanding the LFT defined by $(\Delta \star M)$ as a formal power series; further discussion of formal power series representations and connections to LFT realizations may be found in [13] and [16]. An alternate proof has been given for Lemma 11 using induced 2-norms of $(\Delta \star M)$ rather than a series expansion, and is given in [13] and [17]. The alternate proof is much longer than that presented here but leads more directly to a Kalman-like decomposition structure and to more exact statements on the dimensions of the reduced realizations.

Lemma 12: Suppose

$$
\tilde{X}=\left[\begin{array}{cc}
X & X_{1} \\
X_{1}^{*} & X_{2}
\end{array}\right] \geq 0
$$

and

$$
\tilde{Y}=\left[\begin{array}{cc}
Y & Y_{1} \\
Y_{1}^{*} & Y_{2}
\end{array}\right] \geq 0
$$

where

$$
\operatorname{dim}(X)=\operatorname{dim}(Y)>\operatorname{dim}\left(X_{2}\right)=\operatorname{dim}\left(Y_{2}\right) .
$$

If $\tilde{X} \tilde{Y}=0$, then either $X$ or $Y$ is singular.

Proof: See the Appendix.

We now state and prove the necessity condition.

Theorem 13-Necessity: Suppose the stable system realization $(\boldsymbol{\Delta}, M)$ is given. If there exists a reduced realization $\left(\boldsymbol{\Delta}_{r}, M_{r}\right)$ such that $(\tilde{\Delta} \star \tilde{E})=0$, for all $\tilde{\Delta} \in \mathbf{B}_{\tilde{\Delta}}$, then there exists singular $X \geq 0$ or $Y \geq 0$, both in $\mathcal{T}$, satisfying:

1) $A Y A^{*}-Y+B B^{*} \leq 0$;

2) $A^{*} X A-X+C^{*} C \leq 0$.

Proof: Recall that the difference system $(\tilde{\Delta} \star \tilde{E})=$ $(\Delta \star M)-\left(\Delta_{r} \star M_{r}\right)$ is given by

$$
\tilde{E}=\left[\begin{array}{ccc}
A & 0 & B \\
0 & A_{r} & -B_{r} \\
C & C_{r} & 0
\end{array}\right], \quad \tilde{\Delta}=\left[\begin{array}{cc}
\Delta & 0 \\
0 & \Delta_{r}
\end{array}\right] .
$$

By Lemma 11, if $(\tilde{\Delta} \star \tilde{E})=0$ then there exist $\tilde{X} \geq 0$ and $\tilde{Y} \geq 0$, both in $\tilde{\mathcal{T}}$ satisfying the Lyapunov inequalities for the uncertain system $(\tilde{\boldsymbol{\Delta}}, \tilde{E})$, and $\tilde{X} \tilde{Y}=0$. Since $\tilde{X}$ and $\tilde{Y}$ commute with $\tilde{\Delta} \in \tilde{\boldsymbol{\Delta}}$, they have the structure

$$
\tilde{X}=\left[\begin{array}{cc}
X & X_{1} \\
X_{1}^{*} & X_{2}
\end{array}\right] \text { and } \tilde{Y}=\left[\begin{array}{cc}
Y & Y_{1} \\
Y_{1}^{*} & Y_{2}
\end{array}\right]
$$

where $X$ and $Y$ commute with $\Delta \in \Delta$. Then $X \geq 0$ and $Y \geq 0$ satisfy the Lyapunov inequalities for the uncertain system $(\boldsymbol{\Delta}, M)$, and by Lemma 12 either $X$ or $Y$ is singular.

The results of Theorems 9 and 13 imply that, given an uncertain or multidimensional system representation, if structured singular solutions to either of a pair of LMI's can be found, then an equivalent lower dimension realization exists. Furthermore, if the uncertainty can be properly described by time-varying, or noncommuting, operators on $l_{2}$, then the existence of lower dimension realizations requires such singular LMI solutions. The development of computational methods for solving these types of LMI problems has received extensive attention in the control community (see for example [26]-[28] and the references therein). The fact that we would like to find rank-deficient solutions to these LMI's complicates the computational requirements and the complete set of constraints results in an optimization problem which is not convex. However, a heuristic computational solution has been developed. A brief summary of preliminary test results for this algorithm is presented in the Appendix; for further details see [19] and [20].

\section{A. Minimality}

One notable result which follows immediately from the proof for Theorem 13 is that all minimal realizations for an uncertain system may be obtained by allowable transformations and truncations, where we define minimal as follows.

Definition 14: A realization $(\Delta, M)$ is minimal if $\operatorname{dim}(\Delta)$ is lowest among all equivalent realizations.

The minimality result is stated in the following corollary. 
Corollary 15: Given a stable system realization $(\boldsymbol{\Delta}, M)$, all minimal realizations are found by similarity transformations

$$
\hat{M}=\left[\begin{array}{cc}
T A T^{-1} & T B \\
C T^{-1} & D
\end{array}\right]: \quad T \in \mathcal{T}
$$

and truncations.

If we consider the simplest case for these LFT representations of uncertain systems, that is, there is no uncertainty and $\Delta=\lambda I$, we obtain the standard results, excepting the inequalities in the Lyapunov equations. At the other extreme, if we assume the $\delta_{i}$ are simply noncommuting indeterminates as in the case of formal power series, we do not even have the operator structure for $\Delta$. In this setting, stability and norms have no meaning and indeed are somewhat artificial in the context of pure realization theory. The preceding LMI reducibility results may be simply extended to this case by scaling the $A$ matrix, that is, there will always be some value $\gamma>0$ sufficiently small such that there exists a matrix $Y>0$, $Y \in \mathcal{T}$ satisfying

$$
\gamma^{2} A Y A^{*}-Y<0
$$

The I/O map $(\Delta \star M)$ is then well defined on $l_{2}$ for every

$$
\Delta \in \mathbf{B}_{\gamma} \boldsymbol{\Delta}=\left\{\Delta \in \mathbf{B}_{\Delta}:\|\Delta\|_{l_{2} \rightarrow l_{2}} \leq \gamma\right\}
$$

and solutions $Y \geq 0$ and $X \geq 0$, both in $\mathcal{T}$, can be found satisfying the Lyapunov inequalities formed with the scaled $A$ matrices. If singular $X$ and $Y$ can be found, the realization is reducible as in Theorem 9, with respect to the uncertainty set $\mathbf{B}_{\gamma} \boldsymbol{\Delta}$. These manipulations are interesting mainly because the use of this $\gamma$ scaling illuminates the fact that the reducibility result may be viewed as a topological result, that is, that $\left(\Delta \star M-\Delta_{r} \star M_{r}\right)$ is the zero operator for all operators $\Delta$ in a neighborhood of zero if and only if there exist singular structured solutions to the Lyapunov inequalities.

Alternatively, a subspace, or geometric approach, which does not require stability of the uncertain system realizations may be pursued. This approach is discussed in relation to the results presented herein in [18], where a unified treatment of both the algebraic and geometric perspectives of minimality and decomposition structures for uncertain systems is discussed. Comparisons and connections are outlined showing that the resulting decomposition structures for a given LFT realization are the same, thus showing that standard statespace realization theory may be wholly generalized to LFT realizations for uncertain systems. Additional technical details on the construction of reachable and unobservable subspaces may be found in [29].

\section{CONTROLlability, OBSERVABILITY, AND}

\section{DECOMPOSITION STRUCTURES FOR UNCERTAIN SYSTEMS}

Up to this point, we have presented reducibility conditions for a given realization, $(\boldsymbol{\Delta}, M)$, in terms of structured Gramians, without any discussion of controllability and observability or a direct test for minimality. Naturally, we would like to develop generalizations of the standard controllability and observability matrices and determine the relation these matrices have not only to the structured Gramians, but also to a direct notion of minimality. In this section, we discuss the construction of controllability and observability matrices for uncertain systems modeled by LFT realizations and show that rank conditions on these matrices relate to reducibility of the system realizations and provide for a direct test of minimality. We begin with a discussion of the Kalman-like decomposition structure that results from the proof of Theorem 9 and Lemma 11.

\section{A. Decomposition Structures}

In the proof for Theorem 9, it is made clear that the existence of a singular structured Gramian implies that an equivalent realization can be found which has a Kalman-like decomposition structure. For example, consider the realization

$$
\begin{aligned}
\boldsymbol{\Delta} & =\left\{\operatorname{diag}\left[\delta_{1} I_{n_{1}}, \delta_{2} I_{n_{2}}\right]: \delta_{i} \in \mathcal{L}\left(l_{2}\right)\right\} \\
M & =\left[\begin{array}{ll|r}
A_{11} & A_{12} & B_{1} \\
A_{21} & A_{22} & B_{2} \\
\hline C_{1} & C_{2} & 0
\end{array}\right]
\end{aligned}
$$

and suppose structured Gramians $X=\left[\begin{array}{ll}{ }^{X_{1}} & \\ & X_{2}\end{array}\right] \geq 0$ and $Y=\left[\begin{array}{ll}{ }^{Y_{1}} & \\ { } Y_{2}\end{array}\right] \geq 0$ are found where $X_{1}, X_{2}, Y_{1}$, and $Y_{2}$ are all singular. Then, using an allowable, simultaneously diagonalizing transformation for $X$ and $Y$, we can find an equivalent realization $\hat{M}$ that has the decomposition structure where

$$
\begin{aligned}
\hat{A}_{i j} & =\left[\begin{array}{cccc}
\hat{A}_{i j}^{c o} & 0 & \hat{A}_{i j}^{13} & 0 \\
\hat{A}_{i j}^{21} & \hat{A}_{i j}^{c} & \hat{A}_{i j}^{23} & \hat{A}_{i j}^{24} \\
0 & 0 & \hat{A}_{i j}^{o} & 0 \\
0 & 0 & \hat{A}_{i j}^{43} & \hat{A}_{i j}^{44}
\end{array}\right] \\
B_{i} & =\left[\begin{array}{c}
\hat{B}_{i}^{c o} \\
\hat{B}_{i}^{c} \\
0 \\
0
\end{array}\right], \quad C_{j}=\left[\begin{array}{llll}
\hat{C}_{j}^{c o} & 0 & \hat{C}_{j}^{o} & 0
\end{array}\right]
\end{aligned}
$$

for each $i, j=1,2$.

Similarly, from Theorem 13, we know if $(\Delta, M)$ is reducible, there exist singular structured Gramians for the full realization; these Gramians can be used as above to find a decomposition structure for $(\boldsymbol{\Delta}, M)$.

\section{B. Controllability and Observability Matrices}

If we naively define the controllability and observability matrices, $\mathcal{C}$ and $\mathcal{O}$, as for standard (1-D) state-space realizations, then we cannot directly generalize the relationships between the ranks of $\mathcal{C}$ and $\mathcal{O}$ to reducibility via singular structured Gramians. For example, suppose we take $\mathcal{C}:=$ [ $\left.B A B \cdots A^{N-1} B\right]$, where $N=\sum_{i=1}^{p} n_{i}$. If there exists a singular structured Gramian $Y \geq 0$, then it is easy to see that $\operatorname{rank}(\mathcal{C})<N$ by considering the decomposition structure in the proof of Theorem 9. However, the converse is generally not true. Consider the following example:

and

$$
A=\left[\begin{array}{ll}
-0.110 & 0.040 \\
-0.480 & 0.170
\end{array}\right], \quad B=\left[\begin{array}{l}
0.100 \\
0.300
\end{array}\right]
$$

$$
\boldsymbol{\Delta}=\left\{\left[\begin{array}{ll}
\delta_{1} & \\
& \delta_{2}
\end{array}\right]: \delta_{i} \in \mathcal{L}\left(l_{2}\right)\right\} \text {. }
$$


Then, $\mathcal{C}=\left[\begin{array}{cc}0.100 & 0.001 \\ 0.300 & 0.003\end{array}\right]$ has rank 1 , but there is no singular $Y=\left[\begin{array}{ll}y_{1} & \\ & y_{2}\end{array}\right] \geq 0$ satisfying $A Y A^{*}-Y+B B^{*} \leq 0$.

As a more reasonable generalization of realization theory results associated with controllability and observability matrices for standard state-space models, we propose the following constructions for uncertain systems models, which take into account the inherent structure of the system realization.

Definition 16: Given an uncertain system realization $(\boldsymbol{\Delta}, M)$, where $\boldsymbol{\Delta}$ is structured as in (7), then the controllability matrix is defined as shown in the equation at the bottom of the page. Furthermore, we denote the block rows by $\mathcal{C}_{i}=\left[\begin{array}{lll}B_{i} & A_{i 1} B_{1} & \cdots\end{array}\right]$.

The partitioning of this controllability matrix into block rows is similar to the partitioning proposed for a twodimensional (2-D) system controllability matrices when a relationship to minimality is desired (see [30] and the references therein). However, the block elements of the above controllability matrix are noticeably different than in the 2-D case (or multidimensional case), as the system variables represented by the $\delta_{i}$ in the LFT realizations we consider are assumed to be noncommuting.

The following relation can be shown to hold between singular structured Gramians and rank conditions on $\mathcal{C}$; a sketch of the proof follows.

Lemma 17: Let $(\boldsymbol{\Delta}, M)$ be a stable uncertain system realization, where $\boldsymbol{\Delta}$ is defined as in (1), and suppose $\mathcal{C}$ is the associated structured controllability matrix. Then $\operatorname{rank}\left(\mathcal{C}_{i}\right)<$ $n_{i}$ for some $i=1, \cdots, p$, if and only if there exists a singular $Y \in \mathcal{T}, Y \geq 0$ satisfying $A Y A^{*}-Y+B B^{*} \leq 0$.

Proof-Sketch: Sufficiency is quite straightforward: if there exists a singular structured controllability Gramian $Y$, then using allowable transformations (as in the proof of Theorem 9), we can find a decomposition structure from which it is easy to see that the block rows of the controllability matrix will have reduced rank.

Necessity can be shown using an approach similar to that taken for the proof of Lemma 11 . For each $i=1, \cdots, p$, denote $r_{i}=\operatorname{rank}\left(\mathcal{C}_{i}\right)$, where we assume $r_{i}<n_{i}$ for at least one $i$. Then there exist nonsingular matrices $T_{i} \in \mathbf{C}^{n_{i} \times n_{i}}$ such that

$$
T_{i} \mathcal{C}_{i}=\left[\begin{array}{c}
\tilde{\mathcal{C}}_{i} \\
0
\end{array}\right], \quad \text { where } \tilde{\mathcal{C}}_{i} \text { has } r_{i} \text { rows }
$$

This implies that $T_{i} B_{i}=\left[\begin{array}{c}\tilde{B}_{i} \\ 0\end{array}\right]$, and that $T_{i} A_{i j} B_{j}$ has the form $\left[\begin{array}{c}\times \\ 0\end{array}\right]$, for all $j=1, \cdots, p$. Thus, there exists a $T_{j} \in \mathbf{C}^{n_{j} \times n_{j}}$ such that

$$
T_{i} A_{i j} T_{j}^{-1}=\left[\begin{array}{cc}
\tilde{A}^{11} & \tilde{A}^{12} \\
0 & \tilde{A}^{22}
\end{array}\right] \quad \text { and } \quad T_{j} B_{j}=\left[\begin{array}{c}
\tilde{B}_{j} \\
0
\end{array}\right]
$$

where the zero block of $\tilde{A}_{i j}$ has dimensions $\left(n_{i}-r_{i}\right) \times r_{j}$. Note that if $r_{j}=n_{j}$, then

$$
T_{i} A_{i j} T_{j}^{-1}=\left[\begin{array}{cc}
\tilde{A}^{11} & \tilde{A}^{12} \\
0 & 0
\end{array}\right]
$$

A similar analysis is applied to the $A_{i j} A_{j k} B_{k}$ terms, resulting in an allowable transformation $T=\operatorname{diag}\left[T_{1}, T_{2}, \cdots, T_{p}\right]$, which decomposes the given realization matrix $M$ into an equivalent realization with an uncontrollable-like decomposition structure. We can then construct a singular structured controllability Gramian, $Y$, in the same manner as outlined in the proof of Lemma 11.

The obvious dual definition for the observability matrix can be given, along with the corresponding rank condition which can be proven using a duality argument.

Definition 18: Given an uncertain system realization $(\Delta, M)$, where $\Delta$ is structured as in (1), then the observability matrix is defined by

$$
\mathcal{O}=\left[\begin{array}{cccc}
C_{1} & C_{2} & \cdots & C_{p} \\
C_{1} A_{11} & C_{1} A_{12} & \cdots & C_{1} A_{1 p} \\
\vdots & \vdots & \vdots & \vdots \\
C_{p} A_{p 1} & C_{p} A_{p 2} & \cdots & C_{p} A_{p p} \\
C_{1} A_{11}^{2} & C_{1} A_{11} A_{12} & \cdots & C_{1} A_{11} A_{1 p} \\
\vdots & \vdots & \vdots & \vdots
\end{array}\right] .
$$

Furthermore, we denote the block columns by

$$
\mathcal{O}_{i}=\left[\begin{array}{c}
C_{i} \\
C_{1} A_{1 i} \\
C_{2} A_{2 i} \\
\vdots
\end{array}\right] .
$$

Lemma 19: Let $(\boldsymbol{\Delta}, M)$ be a stable uncertain system realization, where $\boldsymbol{\Delta}$ is defined as in (1), and suppose $\mathcal{O}$ is the associated structured observability matrix. Then $\operatorname{rank}\left(\mathcal{O}_{i}\right)<$ $n_{i}$ for some $i=1, \cdots, p$, if and only if there exists a singular $X \in \mathcal{T}, X \geq 0$ satisfying $A^{*} X A-X+C^{*} C \leq 0$.

From Lemmas 17 and 19, we can directly state the following result connecting minimality to rank deficiencies of the controllability and observability matrices.

Theorem 20: Let $(\boldsymbol{\Delta}, M)$ be a stable uncertain system realization, where $\boldsymbol{\Delta}$ is defined as in (1), and suppose $\mathcal{C}$ and $\mathcal{O}$ are the associated structured controllability and observability matrices. Then $(\boldsymbol{\Delta}, M)$ is a minimal realization if and only if $\operatorname{rank}\left(\mathcal{C}_{i}\right)=n_{i}$ and $\operatorname{rank}\left(\mathcal{O}_{i}\right)=n_{i}$ for every $i=1, \cdots, p$.

These rank tests can be completed on finite dimension controllability and observability matrices, where the maximum dimensions are determined by the number of copies of each $\delta_{i}$ in the structure $\Delta$. Moreover, the degree of singularity of the structured Gramians can be directly related to the difference between the dimension and the rank of $\mathcal{C}_{i}$ and of $\mathcal{O}_{i}$.

$$
\mathcal{C}=\left[\begin{array}{ccccccccc}
B_{1} & A_{11} B_{1} & \cdots & A_{1 p} B_{p} & A_{11}^{2} B_{1} & \cdots & A_{11} A_{1 p} B_{p} & A_{12} A_{21} B_{1} & \cdots \\
B_{2} & A_{21} B_{1} & \cdots & A_{2 p} B_{p} & A_{21} A_{11} B_{1} & \cdots & A_{21} A_{1 p} B_{p} & A_{22} A_{21} B_{1} & \cdots \\
\vdots & \vdots & \vdots & \vdots & \vdots & \vdots & \vdots & \vdots & \vdots \\
B_{p} & A_{p 1} B_{1} & \cdots & A_{p p} B_{p} & A_{p 1} A_{11} B_{1} & \cdots & A_{p 1} A_{1 p} B_{p} & A_{p 2} A_{21} B_{1} & \cdots
\end{array}\right]
$$




\section{Example}

We consider a simple illustrative example with $\Delta$ structure $\operatorname{diag}\left[\delta_{1} I_{2} \delta_{2} I_{2}\right]$. The realization matrices that comprise $M$ are

$$
\begin{aligned}
& A=\left[\begin{array}{l|l}
A_{11} & A_{12} \\
\hline A_{21} & A_{22}
\end{array}\right]=\left[\begin{array}{rr|rc}
0.5 & -0.4 & 0.25 & 0.2 \\
0.05 & 0.2 & 0.15 & 0.05 \\
\hline 0.1 & -0.2 & -0.3 & 0.35 \\
0.2 & -0.4 & -1.0 & 0.9
\end{array}\right] \\
& B=\left[\begin{array}{l}
B_{1} \\
B_{2}
\end{array}\right]=\left[\begin{array}{ll}
1 & 4 \\
0 & 2 \\
\hline 1 & 1 \\
2 & 2
\end{array}\right]
\end{aligned}
$$

and

$$
C=\left[\begin{array}{ll}
C_{1} & C_{2}
\end{array}\right]=\left[\begin{array}{ll|ll}
1 & -2 & 0 & 1
\end{array}\right] .
$$

A simple check of the reducibility of this system can be made by evaluating $\mathcal{C}$ and $\mathcal{O}$, where for example

$$
\mathcal{C}=\left[\begin{array}{cccc}
B_{1} & A_{11} B_{1} & \cdots & A_{12} A_{22} B_{2} \\
\hline B_{2} & A_{21} B_{1} & \cdots & A_{22}^{2} B_{2}
\end{array}\right]
$$

and

$$
\mathcal{O}=\left[\begin{array}{c|c}
C_{1} & C_{2} \\
C_{1} A_{11} & C_{1} A_{12} \\
\vdots & \vdots \\
C_{2} A_{22} A_{21} & C_{2} A_{22}^{2}
\end{array}\right]
$$

suffice. In this case, straightforward rank calculations show that $\operatorname{rank}\left[\mathcal{C}_{1}\right]=2$ and $\operatorname{rank}\left[\mathcal{C}_{2}\right]=1 ; \operatorname{rank}\left[\mathcal{O}_{1}\right]=1$ and $\operatorname{rank}\left[\mathcal{O}_{2}\right]=2$. Thus this system is reducible by dimension 1 in both the first and second uncertainty variables; that is, it can be shown to be unreachable in the $\delta_{2}$ space and unobservable in the $\delta_{1}$ space.

We should also be able to find singular structured Gramians, $X$ and $Y$, that solve the Lyapunov inequalities. Using the LMI toolbox we obtain block-structured solutions with eigenvalues

$$
\lambda(Y)=\left\{\begin{array}{ll|ll}
48.14, & 0.29 & 27.71, & 0
\end{array}\right\}
$$

and

$$
\lambda(X)=\{14.67, \quad 0 \mid 0.82, \quad 8.73\} .
$$

Thus we can find an allowable transformation

$$
T=\left[\begin{array}{ll}
T_{1} & \\
& T_{2}
\end{array}\right]
$$

where $T_{1}$ transforms the first block of $X$, the structured observability Gramian, and $T_{2}$ transforms the second block of $Y$, the structured controllability Gramian, such that

and

$$
\left(T_{1}^{-1}\right)^{*} X_{1} T_{1}^{-1}=\left[\begin{array}{ll}
2.94 & \\
& 0
\end{array}\right]
$$

$$
T_{2} Y_{2} T_{2}^{*}=\left[\begin{array}{ll}
5.54 & \\
& 0
\end{array}\right] \text {. }
$$

Applying this transformation to $\{A, B, C\}$, i.e., computing $\left\{T A T^{-1}, T B, C T^{-1}\right\}$, and truncating the subsystems associated with the zero parts of $X_{1}$ and $Y_{2}$ gives an equivalent lower order realization

$$
A_{r}=\left[\begin{array}{l|l}
0.4 & 0.15 \\
\hline 0.1 & 0.4
\end{array}\right], \quad B_{r}=\left[\begin{array}{ll}
1 & 0 \\
\hline 1 & 1
\end{array}\right]
$$

and

$$
C_{r}=[1 \mid 2] .
$$

Details regarding practical Matlab-based reduction routines may be found in [20].

\section{SUMMARY AND RELATED RESULTS}

In this paper, we have presented a thorough treatment of reducibility of uncertain system realizations based on the existence of structured singular Gramians; related realization theory topics including the definition of and rank tests for structured controllability and observability matrices have also been given. Additional realization theory for uncertain systems has been completed; most closely related is a geometric, or subspace, view of minimality for such systems.

The geometric approach involves decomposing the internal variable space into reachable and unobservable subspaces; this also leads to a Kalman-like decomposition structure for LFT realizations and hence a minimality result. Straightforward generalizations of these reachable and unobservable subspaces for uncertain system realizations are discussed in [18] and [29], where the LFT's are now restricted to be causal operators. An alternate method for constructing a minimal realization and a Kalman-like decomposition structure is given, where the resulting decomposition structure is the same as that in (11). It can be shown that the existence of rank-deficient structured controllability Gramians is directly related to the existence of a nontrivial unreachable subspace in the system state and uncertainty variables; similarly, the existence of rankdeficient structured observability Gramians relates directly to an unobservable subspace [18].

The geometric methods may be applied to realizations that are not stable; however, the procedure relies on finding reduced rank matrices, as does the approach described herein using controllability and observability matrices, which may be numerically ill-conditioned. Note that the LMI-based reducibility results of Section IV may be related to reduction with guaranteed error bounds of uncertain system realizations in the situation where no singular structured Gramians are found, that is, when the reduction is not exact [13], [14]. Although the structured Gramians used in the LMI-based model reduction methods of [14] may be suboptimal, the associated computational problems are easily solved. Model reduction methods for unstable uncertain systems have also recently been developed [31].

State-space synthesis results have also been generalized to uncertain systems: output feedback stabilization for uncertain systems can be reduced via a separation argument to full information and full control problems, which can be solved using LMI's [15]; $H_{\infty}$ optimal control can be generalized to this setting, again with a separation structure and two LMI's with a convex coupling condition [6], [7], [32]. Related notions of stabilizability and detectability for uncertain systems via 
static-state feedback and static output-injection matrices may also be stated [15] and PBH type tests defined [22].

\section{APPENDIX}

\section{A. Proof of Lemma 11}

Consider the formal power series $\mathcal{S}=(\Delta \star M)=D+$ $\sum_{k=0}^{\infty} C \Delta(A \Delta)^{k} B$. We first partition the matrices $A, B$, and $C$ accordingly with the $\Delta$ structure, that is

$$
\begin{aligned}
& A=\left[\begin{array}{ccc}
A_{11} & \cdots & A_{1 p} \\
\vdots & \ddots & \vdots \\
A_{p 1} & \cdots & A_{p p}
\end{array}\right] \\
& B=\left[\begin{array}{c}
B_{1} \\
\vdots \\
B_{p}
\end{array}\right]
\end{aligned}
$$

and

$$
C=\left[\begin{array}{lll}
C_{1} & \cdots & C_{p}
\end{array}\right]
$$

Then, expanding the series $\mathcal{S}$ gives

$$
\begin{aligned}
\mathcal{S}= & D+\sum_{i=1}^{p} C_{i} B_{i} \delta_{i}+\sum_{k=0}^{\infty} \sum_{i_{0}, \cdots, i_{k}=1}^{p} \\
& \cdot C_{i_{k}} A_{i_{k}, i_{k-1}} \cdots A_{i_{1} i_{0}} B_{i_{0}} \delta_{i_{k}} \cdots \delta_{1_{0}}
\end{aligned}
$$

where, by assumption, $\mathcal{S}=0$.

Since the $\delta_{i}$ are noncommuting, $\mathcal{S}$ is identically zero if and only if each coefficient in the series is zero, that is

$$
D=0, \quad C_{i} B_{i}=0, C_{i} A_{i j} B_{j}=0, \quad C_{i} A_{i j} A_{j k} B_{k}=0, \cdots
$$

for every $i, j, k=1, \cdots, p$. We consider each set of terms separately and show that the given realization may be transformed to one having a particular decomposition structure, similar to the Kalman decomposition structure for 1-D systems.

First, consider the $C_{i} B_{i}$ terms: $C_{i} B_{i}=0$ if and only if there exists $T_{i}$ nonsingular, such that

$$
C_{i} T_{i}^{-1}=\left[\begin{array}{lll}
0 & 0 & \tilde{C}
\end{array}\right]_{i} \text { and } T_{i} B_{i}=\left[\begin{array}{c}
\tilde{B} \\
0 \\
0
\end{array}\right]_{i}
$$

where $\tilde{C}_{i}$ has full column rank, $\tilde{B}_{i}$ has full row rank, and the submatrices of $C_{i}$ and $B_{i}$ are equivalently partitioned, for each $i, j=1, \cdots, p$. We will henceforth absorb all such transformations and assume the realization matrices are already structured into zero and nonzero block submatrices. We then partition each $A_{i j}$ accordingly with the partitions of $C_{i}$ and $B_{j}$.

Consider the $C_{i} A_{i j} B_{j}$ terms

$$
\begin{aligned}
C_{i} A_{i j} B_{j} & =\left[\begin{array}{lll}
0 & 0 & \tilde{C}
\end{array}\right]_{i}\left[\begin{array}{lll}
A^{11} & A^{12} & A^{13} \\
A^{21} & A^{22} & A^{23} \\
A^{31} & A^{32} & A^{33}
\end{array}\right]_{i j}\left[\begin{array}{c}
\tilde{B} \\
0 \\
0
\end{array}\right]_{j} \\
& =\tilde{C}_{i} A_{i j}^{31} \tilde{B}_{j}=0 .
\end{aligned}
$$

Since $\tilde{C}_{i}$ and $\tilde{B}_{j}$ are both full rank, this implies that $A_{i j}^{31}=0$.
Next consider the $C_{i} A_{i j} A_{j l} B_{l}$ terms

$$
\begin{gathered}
C_{i} A_{i j} A_{j l} B_{l}=\left[\begin{array}{lll}
0 & \tilde{C}_{i} A_{i j}^{32} & \tilde{C}_{i} A_{i j}^{33}
\end{array}\right]\left[\begin{array}{c}
A_{j l}^{11} \tilde{B}_{l} \\
A_{j l}^{21} \tilde{B}_{l} \\
0
\end{array}\right]=0 \\
\text { if and only if } A_{i j}^{32} A_{j l}^{21}=0 .
\end{gathered}
$$

As with the $C_{i} B_{i}$ terms, we can transform $A_{i j}^{32}$ to $\left[\begin{array}{lll}0 & 0 & \tilde{A}_{i j}^{32}\end{array}\right]$ and $A_{j l}^{21}$ to

$$
\left[\begin{array}{c}
\tilde{A}_{j l}^{21} \\
0 \\
0
\end{array}\right]
$$

where $\tilde{A}_{i j}^{32}$ has full column rank, $\tilde{A}_{j l}^{21}$ has full row rank, and the submatrices of $A_{i j}^{32}$ and $A_{j l}^{21}$ are equivalently partitioned. We now have the following decomposition structure for each $A_{i j}$ :

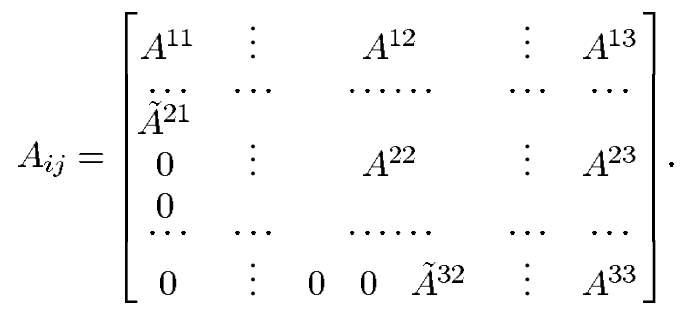

Note that across each block row, indexed by for example $i$, the $A^{21}$ blocks in the set of submatrices $\left\{A_{i j}\right\}_{j=1}^{p}$ are equivalently partitioned; similarly, down each block column indexed by $j$, the $A^{32}$ blocks in the set of submatrices $\left\{A_{i j}\right\}_{i=1}^{p}$ are also equivalently partitioned.

Evaluating the next few sets of series coefficients, $C_{i} A_{i j} A_{j l} A_{l w} B_{w}$, etc., we obtain the same decomposition structure for each of the $A_{i j}^{22}$ blocks as that in (13). This process is repeated for a finite number of series coefficients, leading to a decomposition structure for each $C_{j}, A_{i j}, B_{i}$ subsystem; this finite number is determined by the number of variables, $p$, and the dimensions of the realization matrices. As an example, for $p=2$, the resulting decomposition can be generally written as follows:

$$
M=\left[\begin{array}{ccccc|c}
\hat{A}_{11}^{11} & \hat{A}_{11}^{12} & \vdots & \hat{A}_{12}^{11} & \hat{A}_{12}^{12} & \hat{B}_{1} \\
0 & \hat{A}_{11}^{22} & \vdots & 0 & \hat{A}_{12}^{22} & 0 \\
\cdots & \cdots & \cdots & \cdots & \cdots & \cdots \\
\hat{A}_{21}^{11} & \hat{A}_{21}^{12} & \vdots & \hat{A}_{22}^{11} & \hat{A}_{22}^{12} & \hat{B}_{2} \\
0 & \hat{A}_{21}^{22} & \vdots & 0 & \hat{A}_{22}^{22} & 0 \\
\hline 0 & \hat{C}_{1} & \vdots & 0 & \hat{C}_{2} & 0
\end{array}\right] .
$$

Note that the matrix partitions in (14) (denoted by "'s) do not necessarily correspond to the previous partitions (denoted by "s). Note also that the above partitions are constructed such that the $\hat{A}_{i i}^{11}$ and $\hat{A}_{i i}^{22}$ submatrices are square, and the lower left submatrix of each $\hat{A}_{i i}$, which is identically zero, has the largest dimensions possible.

Applying Lemma 8 and carrying out the matrix multiplications, it is straightforward to see there exist structured singular 
TABLE I

Preliminary Trace Algorithm Results

\begin{tabular}{c|c}
\hline$\eta$ Upper Bound & $\%$ Models Reducible \\
\hline \hline $5 \times 10^{-5}$ & $100 \%$ \\
\hline $1 \times 10^{-6}$ & $86 \%$ \\
\hline $1 \times 10^{-10}$ & $77 \%$ \\
\hline
\end{tabular}

matrices

and

$$
\hat{X}=\operatorname{diag}\left[0, \hat{X}_{1}^{22}, 0, \hat{X}_{2}^{22}, \cdots, 0, \hat{X}_{p}^{22}\right] \geq 0
$$

$$
\hat{Y}=\operatorname{diag}\left[\hat{Y}_{1}^{11}, 0, \hat{Y}_{2}^{11}, 0, \cdots, \hat{Y}_{p}^{11}, 0\right] \geq 0
$$

both in $\mathcal{T}$, satisfying

$$
A \hat{Y} A^{*}-\hat{Y} \leq 0 \text { and } A^{*} \hat{X} A-\hat{X} \leq 0 .
$$

Furthermore, scaling $\hat{Y}$ and $\hat{X}$ by constants, as necessary, gives $X \geq 0$ and $Y \geq 0$ both in $\mathcal{T}$, such that conditions 1) and 2) are satisfied, and clearly $X Y=0$.

\section{B. Proof of Lemma 12}

Let $\operatorname{dim}(X)=\operatorname{dim}(Y)=n \times n$ and $\operatorname{dim}\left(X_{2}\right)=$ $\operatorname{dim}\left(Y_{2}\right)=k \times k$, where $k<n$. By contradiction, suppose both $X$ and $Y$ are nonsingular. Then $X Y$ is nonsingular and $\operatorname{rank}(X Y)=n$.

Note that $\tilde{X} \tilde{Y}=0$ implies that $X Y=-X_{1} Y_{1}^{*}$, thus $\operatorname{rank}\left(X_{1} Y_{1}^{*}\right)=n$, where $\operatorname{dim}\left(X_{1}\right)=n \times k$ and $\operatorname{dim}\left(Y_{1}^{*}\right)=$ $k \times n$. However,

$$
\operatorname{rank}\left(X_{1} Y_{1}^{*}\right) \leq \min \left\{\operatorname{rank}\left(X_{1}\right), \operatorname{rank}\left(Y_{1}^{*}\right)\right\} \leq k<n .
$$

Thus $X$ or $Y$ is singular and $\operatorname{rank}(X Y) \leq k$.

\section{Overview of Computational Results}

Although feasible solutions to the Lyapunov inequalities may easily be computed using convex programming methods, the reducibility problem considered in this paper is a reduced rank LMI problem. Unfortunately, reduced rank LMI problems result in neither convex nor quasiconvex optimization problems, thus we cannot directly apply existing LMI techniques to obtain optimal solutions. However, LMI methods have been used in heuristic algorithms to obtain suboptimal solutions to reduced rank LMI problems. The following simple algorithm has been used to compute near-singular solutions to the system Lyapunov inequalities.

Given an uncertain system realization $(\Delta, M)$ find

$$
\begin{array}{r}
\circ \min _{Y} \operatorname{Trace}(Y): A Y A^{*}-Y+B B^{*} \leq 0, \\
Y \geq 0 \text { and } Y \in \mathcal{T}
\end{array}
$$

and

$$
\begin{array}{r}
\circ \min _{X} \operatorname{Trace}(X): A^{*} X A-X+C^{*} C \leq 0, \\
X \geq 0 \text { and } X \in \mathcal{T} .
\end{array}
$$

Preliminary tests of this algorithm have been completed using the LMI Toolbox [26]: 20 multidimensional system realizations, each with two to five transform/uncertainty variables and dimensions ranging from 5 to 15 have been constructed and tested. These realizations have been constructed to be exactly reducible, that is, for each realization there exist singular structured matrices $X$ and $Y$ satisfying the associated Lyapunov inequalities. Evaluation of the Trace algorithm on the test realizations is based on the eigenvalues of the resulting LMI solutions $X$ and $Y$. Specifically, we consider the ratio, denoted by $\eta$, of the largest "zero value" eigenvalue to the smallest nonzero valued eigenvalue. So, for example, if $\operatorname{eig}(X)=\left\{1.000,0.724,0.711,0.531,1.000 \times 10^{-6}\right\}$, then $\eta=5.31 \times 10^{-5}$. Note that the same solutions $X$ and $Y$ may always be used to determine guaranteed error bounds on the reduction, as described in [14].

As we know a priori the dimensions that may be reduced with no error for each test case, we are then able to determine the success or failure of this algorithm. The results based on three different criteria for $\eta$ are given in Table I.

\section{REFERENCES}

[1] J. C. Doyle, "Analysis of feedback systems with structured uncertainties," Proc. Inst. Elec. Eng., pt. D, pp. 242-250, 1982.

[2] M. Safonov, "Stability margins of diagonally perturbed multivariable feedback systems," Proc. Inst. Elec. Eng., pt. D, pp. 251-256, 1982.

[3] _ Stability and Robustness of Multivariable Feedback Systems. Cambridge, MA: MIT Press, 1980.

[4] K. Zhou, J. C. Doyle, and K. Glover, Robust and Optimal Control. Englewood Cliffs, NJ: Prentice-Hall, 1996.

[5] G. Dullerud, Control of Uncertain Sampled-Data Systems. New York: Birkhauser, 1995

[6] P. Apkarian and P. Gahinet, "A convex characterization of gainscheduled $H$-infinity controllers," IEEE Trans. Automat. Contr., vol. 40, 1995.

[7] A. Packard, "Gain scheduling via linear fraction transformations," Syst. Contr. Lett., pp. 79-92, 1994.

[8] J. Shamma, "Robust stability with time-varying structured uncertainty," IEEE Trans. Automat. Contr., vol. 39, Apr. 1994.

[9] P. M. Young, "Robustness with parametric and dynamic uncertainty," Ph.D. dissertation, California Inst. Technol., 1993.

[10] A. Packard and J. C. Doyle, "The complex structured singular value," Automatica, vol. 29, no. 1, pp. 71-109, 1993.

[11] A. Megretski and S. Treil, "S-procedure and power distribution inequalities: A new method in optimization and robustness of uncertain systems," Mittag-Leffler Inst., 1991.

[12] F. Paganini, "Sets and constraints in the analysis of uncertain systems," Ph.D. dissertation, California Inst. Technol., Dec. 1995.

[13] C. L. Beck, "Model reduction and minimality for uncertain systems," Ph.D. dissertation, California Inst. Technol., Jan. 1996

[14] C. L Beck, J. C. Doyle, and K. Glover, "Model reduction of multidimensional and uncertain systems," IEEE Trans. Automat. Contr., vol. 41, Oct. 1996.

[15] W.-M. Lu, K. Zhou, and J. C. Doyle, "Stabilization of uncertain linear systems and linear multi-dimensional systems," IEEE Trans. Automat. Contr., vol. 41, Jan. 1996

[16] C. L. Beck and J. C. Doyle, "Realizations of uncertain systems and formal power series," in Proc. American Control Conf., 1995.

[17] C. L. Beck, "Minimality for uncertain systems and IQC's," in Proc. IEEE Control and Decision Conf., 1994, pp. 3068-3074.

[18] C. Beck and R. D'Andrea, "Minimality, controllability and observability for uncertain systems," in Proc. American Control Conf., 1997, pp. 3130-3135.

[19] P. Bendotti and C. L. Beck, "On the role of LFT model reduction methods in robust controller synthesis for a pressurized water reactor," IEEE Trans. Contr. Syst. Technol., vol. 7, Mar. 1999.

[20] C. Beck and R. D'Andrea, "Computational study and comparisons of LFT reducibility methods," in Proc. American Control Conf., 1998.

[21] J. Berstel and C. Reutenauer, Rational Series and Their Languages (Monographs on Theoretical Computer Science). Springer-Verlag, 1988

[22] F. Paganini and J. C. Doyle, "Analysis of implicitly defined systems," in Proc. IEEE Control and Decision Conf., 1994, pp. 3673-3678.

[23] A. Megretski, "Necessary and sufficient conditions of stability: A multiloop generalization of the circle criterion," IEEE Trans. Automat. Contr., 1993. 
[24] R. Braatz, P. Young, J. C. Doyle, and M. Morari, "Computational complexity of $\mu$ calculation," IEEE Trans. Automat. Contr., pp. 1000-1002, 1994.

[25] O. Toker, "Complexity issues in system theory and solution procedures for certain robust control problems," Ph.D. dissertation, Ohio State Univ., 1995.

[26] P. Gahinet, A. Nemirovskii, A. J. Laub, and M. Chilali, The LMI Control Toolbox. Natick, MA: MathWorks, 1995.

[27] F. Alizadeh, J. P. Haeberly, M. Nayakkankuppam, and M. Overton, SDPpack: New Code for Semidefinite Programming, 1997, available via http://www.cs.nyu.edu/phd_students/madhu/sdppack/sdppack.html.

[28] S. Boyd, L. E. Ghaoui, E. Feron, and V. Balakrishnan, Linear Matrix Inequalities in Systems and Control Theory. Philadelphia, PA: SIAM, 1994.

[29] R. D'Andrea and S. Khatri, "Kalman decomposition of linear fractional transformation representations and minimality," in Proc. American Control Conf., 1997, pp. 3557-3561.

[30] N. K. Bose, Applied Multidimensional Systems Theory. New York: Van Nostrand Reinhold, 1982.

[31] C. L. Beck and P. Bendotti, "Model reduction methods for unstable uncertain system," in Proc. IEEE Control and Decision Conf., 1997, pp. $3298-3303$.

[32] G. Scorletti and L. E. Ghaoui, "Improved linear matrix inequality conditions for gain-scheduling," in Proc. IEEE Control and Decision Conf., 1995.

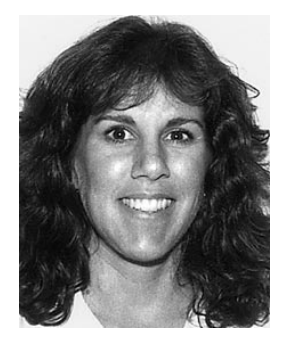

Carolyn L. Beck (S'94-A'96) received the B.S. degree from California State Polytechnic University, Pomona, in 1984 and the M.S. degree from Carnegie Mellon University, Pittsburgh, PA, in 1985, in electrical and computer engineering. She received the $\mathrm{Ph} . \mathrm{D}$. degree in electrical engineering from the California Institute of Technology, Pasadena, in 1996.

From 1985 through 1989, she was employed as a Research and Development Engineer for HewlettPackard in Santa Clara, CA. From January 1996 through June 1996, she was a Postdoctoral Research Assistant at Lund Institute of Technology in Sweden. She joined the Department of Electrical Engineering at the University of Pittsburgh in September 1996 where she was an Assistant Professor until July 1999. She is currently an Assistant Professor at the University of Illinois at Urbana-Champaign in the Department of General Engineering. Her research interests include the development of modeling and control methods for complex systems, with applications in power systems and bioengineering.

Dr. Beck received a National Science Foundation CAREER Award in 1998.

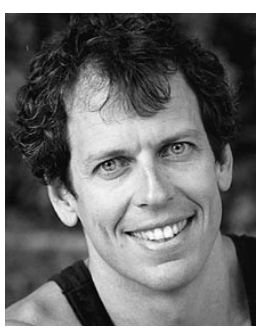

John Doyle (M'96) received the B.S. and M.S. degrees in electrical engineering from MIT and a $\mathrm{Ph} . \mathrm{D}$. degree in mathematics from the University of California, Berkeley.

$\mathrm{He}$ is now a Professor in Control and Dynamical Systems at the California Institute of Technology, Pasadena. His research interests include robustness of complex systems.

Dr. Doyle has received several prize paper awards, including two Axelby and a Baker Prize from the IEEE. 